\title{
A modified murine model based on hydrodynamic injection for the analysis of chronic human hepatitis B virus infection
}

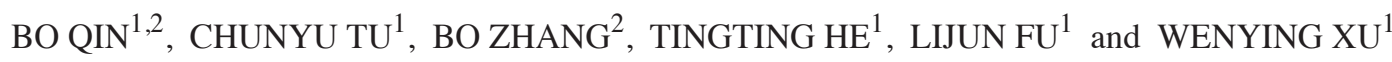 \\ ${ }^{1}$ Shaoxing Center for Disease Control and Prevention, Shaoxing, Zhejiang; ${ }^{2}$ State Key Laboratory of Virology, \\ Wuhan Institute of Virology, Chinese Academy of Sciences, Wuhan, Hubei, P.R. China
}

Received April 18, 2013; Accepted September 26, 2013

DOI: $10.3892 / \mathrm{mmr} .2013 .1732$

\begin{abstract}
Hepatitis B virus (HBV) is a persistent pathogen that causes acute and chronic necroinflammatory liver disease and is attributable to $\sim 1$ million deaths per year. In the present study, a conventional murine model was introduced based on the hydrodynamic injection of engineered replication-competent HBV DNA into the tail veins of C57BL/6 mice. In a previous study, nine in-frame ATG (start) codons in the $\mathrm{S}$ open reading frame (S1-S9) were analyzed. The highly conserved ATG S5 was mutated to ACG by T378C, which led to the substitution sM75T and inhibition of the production and secretion of the hepatitis B surface antigen (HBsAg), and subsequent inhibition of HBV replication. In the present study, T378C was introduced into the pAAV-HBV1.3 plasmid and was confirmed to affect HBsAg production and secretion, and HBV replication in vivo, which was in agreement with the previous in vitro results. Furthermore, the murine model was improved by co-injection of the replication-competent HBV plasmid DNA with Lipofectamine 2000 (LP). In this model, LP not only significantly enhanced HBV replication in mice, but also upregulated the expression of HBsAg and the hepatitis B core antigen. The current modified murine model was superior to the conventional murine HBV model based on HBV challenge by hydrodynamic injection.
\end{abstract}

\section{Introduction}

Approximately 2 billion people worldwide are infected with human hepatitis B virus (HBV) (1) and $>350$ million are chronically infected and at high risk of progression to cirrhosis, liver failure or hepatocellular carcinoma $(2,3)$. More than half of all liver cancers are attributable to HBV infection;

Correspondence to: Dr Bo Qin, Shaoxing Center for Disease Control and Prevention, New Century Street, Paojiang, Shaoxing, Zhejiang 312071, P.R. China

E-mail: qinbo0809@hotmail.com

Key words: hepatitis B virus, Lipofectamine 2000, hydrodynamic injection, immunohistochemical staining, Southern blot analysis and HBV-related liver diseases remain a major public health concern, leading to $\sim 1$ million deaths per year (3).

Advances in established cost-effective and reliable experimental models have accelerated the development of therapeutic modalities for these life-threatening viral infections (3). Although progress has been made, in vitro and in vivo HBV assays are being developed, but are impeded by a lack of understanding of HBV specificity for and entry into human liver cells, as well as reliable cell and animal infection models (4). In addition to humans, only chimpanzees and a small mammal, the treeshrew (families Tupaiidae and Ptilocercidae), are known to be susceptible to human HBV infection (5-7). Previously, basic studies of HBV and the development of antiviral therapeutics were hindered by the lack of suitable in vitro infection systems and animal models (8). Currently, only suboptimal cell transfection- and murine hydrodynamic injection (HI)-based assays are available for $\mathrm{HBV}$ research (9).

To analyze clinical HBV replication, isolates are obtained by the transfection of replicative recombinant HBV DNA into hepatoma cell lines or HI of replication-competent replicons into the tail veins of C57BL/6 mice which are able to replicate and secrete HBV virions $(10,11)$. Previously, in order to obtain a HBV murine model, $10 \mathrm{mg}$ HBV plasmid DNA was injected into the tail veins of mice in a volume of phosphate-buffered saline equivalent to $8 \%$ of the mouse body weight, with the total volume being delivered within 5-8 sec (12-14). In a previous study, the plasmid, pAAV-HBV1.3, did not replicate properly in mice, thus it was difficult to detect HBV DNA in the liver and serum (14). The HBV core antigen (HBcAg) was challenging to detect, particularly in HBV mutants with weaker replication capacity compared with wild-type (WT) HBV (14). In order to enhance HBV replication in mice, $10 \mathrm{mg}$ plasmid pAAV-HBV1.3 and $20 \mu 1$ Lipofectamine 2000 (LP) transfection reagent (Invitrogen Life Technologies, Carlsbad, $\mathrm{CA}$, USA) were mixed and administered via $\mathrm{HI}$ into the tail veins of C57BL/6 mice. Plasmid DNA is well distributed and packaged with LP in liposomes, which facilitates the transfer of DNA to the murine liver cells. The optimized murine HBV-LP model was superior to the murine HBV model without LP based on HBV challenge via HI in mice, at the DNA and protein levels.

HBV has a 3.2-kb, relaxed circular (RC), partially double-stranded overlapped DNA genome containing four overlapping open reading frames (ORFs): P, S, C and X $(15,16)$. 
HBV possesses the smallest known DNA viral genome, the latter of which optimized genomic organization through an overlapping gene strategy (17). The S ORF encodes the surface proteins, L, M and S (the HBsAg), through alternative translation initiation from three in-frame start codons (18). In our previous study, a methionine (M) to threonine (T) substitution at residue 75 (sM75T) was found to be associated with reduced $\mathrm{HBV}$ replication and HBsAg expression in vitro. In the present study, the previously modified HBV murine model was used to determine whether sM75T influenced HBV replication and HBsAg expression in vitro similar to that in vivo. The results obtained were as expected.

\section{Materials and methods}

Cell culture and treatment. Huh7 human hepatocarcinoma cells were cultured in Dulbecco's modified Eagle's medium (DMEM; Invitrogen, Carlsbad, CA, USA) at $37^{\circ} \mathrm{C}$ in a $5 \%$ $\mathrm{CO}_{2}$ atmosphere supplemented with $10 \%$ fetal bovine serum (Gibco-BRL, Carlsbad, CA, USA), $2 \mathrm{mM} / 1$ glutamine, $100 \mathrm{IU} / \mathrm{ml}$ penicillin, and $100 \mathrm{IU} / \mathrm{ml}$ streptomycin. Huh7 cells were seeded in 6-well plates and transfected with $2 \mu \mathrm{g}$ plasmids per well using LP (19). Female C57BL/6 (H-2b) mice (age, 6-8 weeks) were raised under specific pathogen-free conditions in the Central Animal Laboratory of Shaoxing Centre for Disease Control and Prevention (Shaoxing, China) and were handled following animal ethics guideline standards, according to the principles of animal protection, animal welfare and ethical inspection. The study was approved by the Medical Laboratory Animal Management Committee of Zhejiang Province (Department of Health of Zhejiang Province, Zhejiang, China). C57BL/6 mice were challenged with HBV replication-competent plasmids with or without LP administered by HI. The mouse assay was repeated three times, six mice were used in each group and there were seven groups in total: negative control, pBSK-HBV1.3, pAAV-HBV1.3, pAAV-HBV1.3-LP, pBSK-HBV1.3-T378C, pAAV-HBV1.3 T378C and pAAV-HBV1.3- T378C-LP.

Plasmid constructs. HBV mutants were constructed using fusion polymerase chain reaction (PCR), with primers carrying aimed mutations and WT pBSK-HBV1.3 as a template, which is a replication-competent plasmid 1.3-fold greater in length than the HBV genome. The sM75T was included in the pBSK-HBV1.3 plasmid to construct pBSK-HBV1.3-sM75T in our previous study (19). For the in vivo assay, pAAV-HBV-1.3 and pAAV-HBV1.3-rtXs were constructed based on pHBV1.3 and pHBV1.3-rtXs, respectively. pHBV1.3-WT/MT and pAAV were digested with the $S a c I$ and $S a c I I$ restriction endonuclease (Takara Bio, Dalian, China), then end-filled with T4 and Klenow DNA polymerase (Takara Bio), respectively (14). The recovered products were digested by HindIII (Takara Bio) and ligated by T4 ligase (New England BioLabs, Beijing, China) to generate pAAV-HBV1.3 and pAAV-sM75T. All primers used in our study were manufactured by Sangon Biotech (Shanghai, China).

Enzyme-linked immunosorbent assay (ELISA). Huh7 cells were transfected with the indicated HBV-bearing plasmids and the hepatitis B surface antigen (HBsAg) and the hepatitis B e antigen ( $\mathrm{HBeAg})$ in the supernatant at $96 \mathrm{~h}$ post-transfection (hpt) and were detected using an ELISA kit for the detection of HBsAg/HBeAg (Shanghai Kehua Diagnostic Medical Products Co., Ltd., Shanghai, China), according to the manufacturer's instructions (20).

$q P C R$. When the cell lysis solution had been treated with DNase I (Roche Applied Science, Penzberg, Germany) at $37^{\circ} \mathrm{C}$ for $30 \mathrm{~min}$ to digest the plasmid DNA, total HBV DNA was purified from the lysates of Huh7 cells $96 \mathrm{hpt}$ and used as the template for qPCR which was conducted using SYBR-Green I (Roche, Mannheim, Germany) on a Light Cycler real-time PCR unit (Applied Biosystems, Inc., Foster City, CA, USA) according to the manufacturer's instructions. Primers (sense: 5'-GTTGCCCGTTTGTCCTCTAATTC-3' and antisense: 5'-GGAGGGATACATAGAGGTTCCTT-3' for RC) hybridized to the HBV surface gene were designed to quantify the HBV DNA RC genomes (100-bp fragments) by qPCR relative to an external plasmid DNA standard.

Analysis of encapsidated HBV DNA. Replication-competent HBV WT and mutant-type (MT) plasmids were transfected into Huh7 cells or injected into C57BL/6 mice, which were previously challenged via $\mathrm{HI}$ of replication-competent pAAV-HBV1.3 or -T378C, respectively, with or without LP, as described previously (12). HBV DNA replicative intermediates from intracellular core particles were extracted and subjected to agarose gel electrophoresis, followed by denaturation and southern blotting with a ${ }^{32} \mathrm{P}$-labeled full-length HBV probe, as described previously (14). Hybridization signals were visualized and analyzed using a phosphoimager (Cyclone ${ }^{\circledR}$ Plus Storage Phosphor System; Parkard Instrument Company, Inc., Meriden, CT, USA). Data from the densitometric analyses were quantified using OptiQuant software (PerkinElmer, Inc., Waltham, MA, USA).

Immunohistochemical staining. Liver tissues were collected from mice sacrificed at the indicated time points. Intrahepatic $\mathrm{HBcAg}$ of mice was visualized by immunohistochemical staining of formalin-fixed paraffin-embedded tissues by rabbit anti-HBc antibodies (DAKO) and secondary goat antirabbit IgG HRP antibody (DAKO), using an and Envision System (DAKO). The Envision system is also know as the enhanced labeled polymer system. The liver sections were also stained with hematoxylin and eosin $(12,14)$.

Statistical analysis. The statistical analysis was conducted using GraphPad (GraphPad software, San Diego, CA, USA). Differences in multiple comparisons were determined for statistical significance using the Student's t-test. $\mathrm{P}<0.05$ was considered to indicate a statistically significant difference. Results are presented as the mean \pm SD.

\section{Results}

Replication and protein expression of WT and MT plasmids. The plasmid pBSK-HBV1.3 bearing a replication-competent WT HBV, which was 1.3-fold greater in length than the genotype A genome (GenBank accession no. U95551, ayw), was used as a backbone to construct mutant pBSK-HBV1.3. 


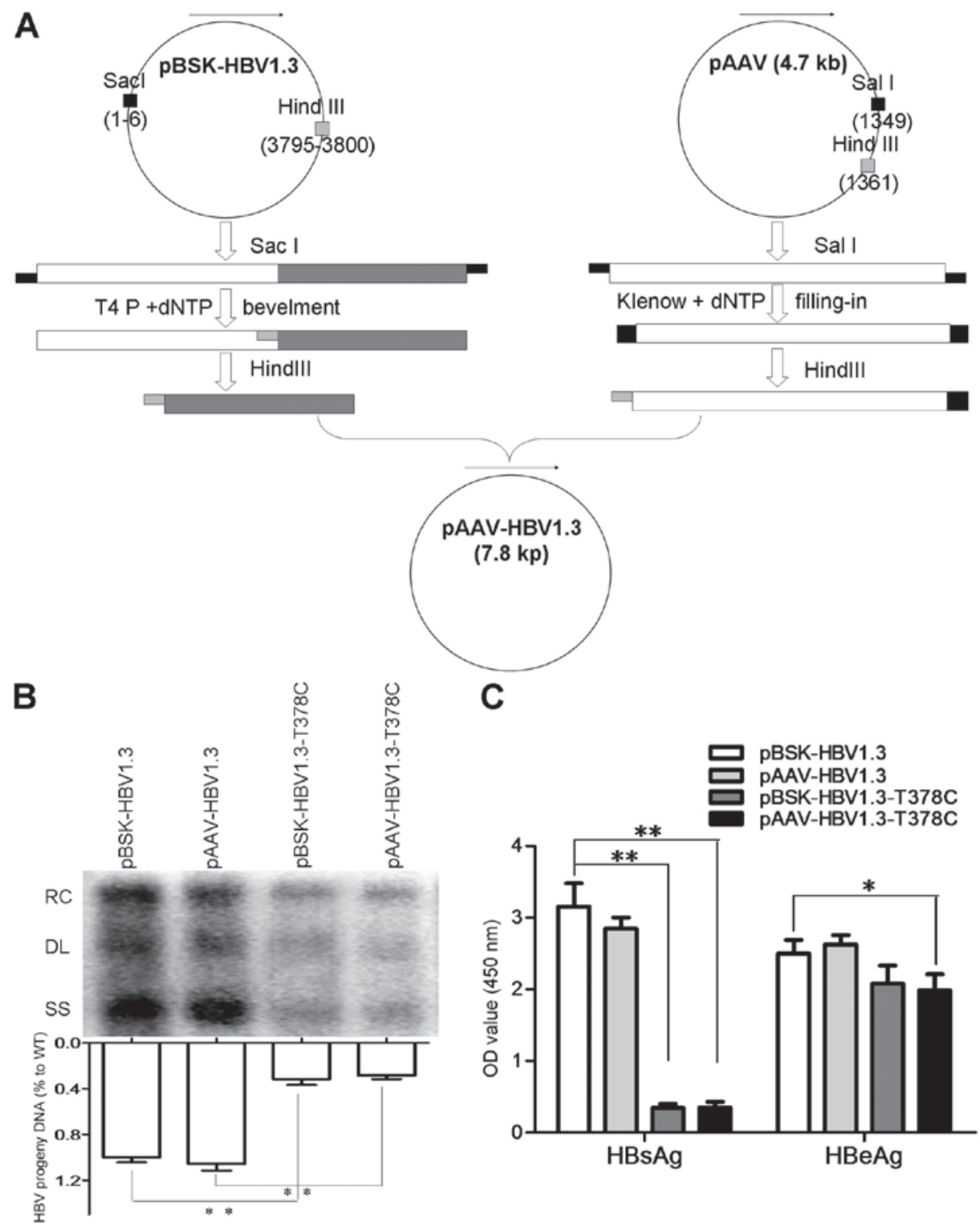

Figure 1. Construction strategy of pAAV-HBV1.3, replication and protein expression of WT and MT plasmids. (A) For the in vivo assay, pAAV-HBV-1.3 and pAAV-HBV1.3-T378C were constructed based on pHBV1.3 and pHBV1.3-T378C plasmids, respectively. The pHBV1.3/pHBV1.3-T378C and pAAV plasmids were digested with $S a c I$, then end-filled with T4 and Klenow DNA polymerase, respectively. The recovered products were digested with HindIII and ligated by T4 ligase to generate pAAV-HBV-1.3 or pAAV-HBV1.3-T378C. (B) Detection of HBV replication intermediates by southern blot analysis. HBV replication RC, DL, and SS HBV DNAs are indicated (upper panel). Intracellular encapsidated HBV DNA levels of each construct were compared with that of the WT genome (set as 100\%, lower panel). (C) The expression of HBsAg and HBeAg were measured using commercial enzyme-linked immunosorbent assay kits. Each value is presented as the mean of three independent experiments. The error bars represent the standard deviation. ${ }^{*} \mathrm{P}<0.05$ and ${ }^{* *} \mathrm{P}<0.01$. HBV, hepatitis $\mathrm{B}$ virus; WT, wild-type; MT, mutant-type; RC, relaxed circular; DL, double-stranded linear; SS, single-stranded; HBsAg, hepatitis B surface antigen; HBeAG, hepatitis B e antigen.

The sM75T in the $s$ gene was introduced into pBSK-HBV1.3 to obtain pBSK-HBV1.3-sM75T. For the in vivo assay, pAAV-HBV-1.3 and pAAV-HBV1.3-sM75T were constructed based on plasmids pBSK-HBV1.3 and pBSK-HBV1.3-sM75T, respectively, as described above (Fig. 1A). Plasmids pBSK-HBV1.3, pBSK-HBV1.3-sM75T, pAAV-HBV1.3 and pAAV-HBV1.3-sM75T were transfected into Huh7 cells and the HBsAg and $\mathrm{HBeAg}$ in the culture medium were detected by ELISA. Replicative HBV intermediates from intracellular core particles were extracted from Huh7 cells and detected by southern blot analysis, as previously described (14). The data indicated that pBSK-HBV1.3 and pAAV-HBV1.3 had similar replication levels of HBs/eAg, while pBSK-HBV1.3-sM75T and pAAV-HBV1.3-sM75T had similar replication levels to HBs/eAg. In addition, the pBSK-HBV1.3-sM75T and pAAV-HBV1.3-sM75T exhibited moderately decreased levels of HBV DNA and HBeAg compared with the WT and a markedly decreased expression level of HBsAg (Fig. 1B and C).

$L P$ enhances $H B V$ replication in mice. The LP transfection reagent is a proprietary formulation for transfecting nucleic acids (DNA, RNA and mRNA) into a wide range of eukaryotic cells and was used in the present study to package HBV plasmid DNA allowing entry into the Huh7 cells. However, it is not clear whether LP promotes HBV replication when mixed and co-injected with pAAV-HBV1.3 into 
A

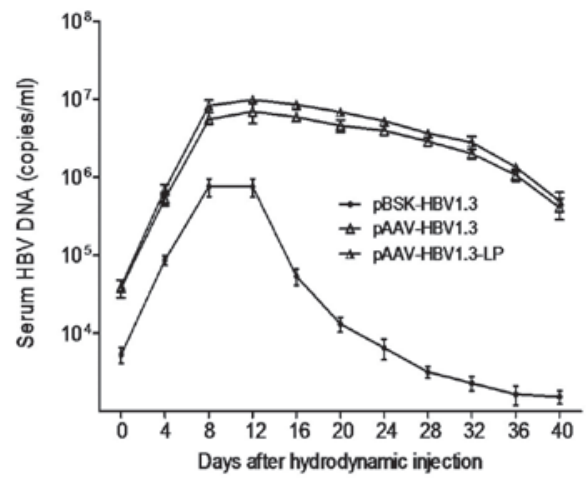

B

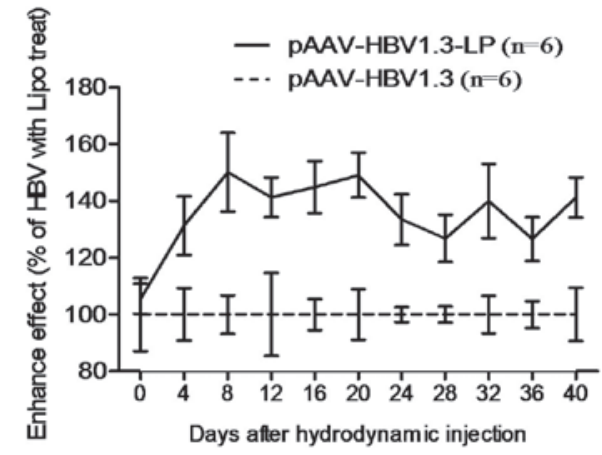

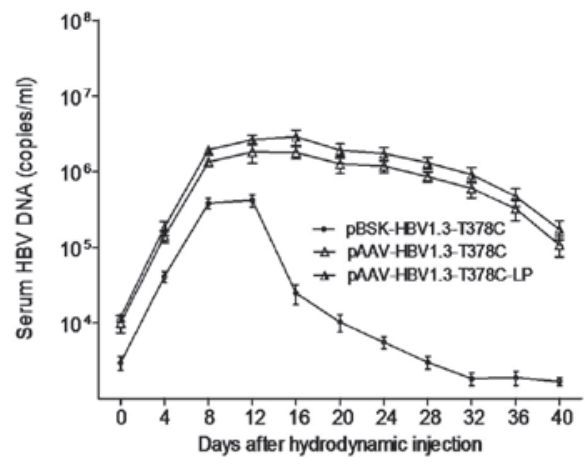

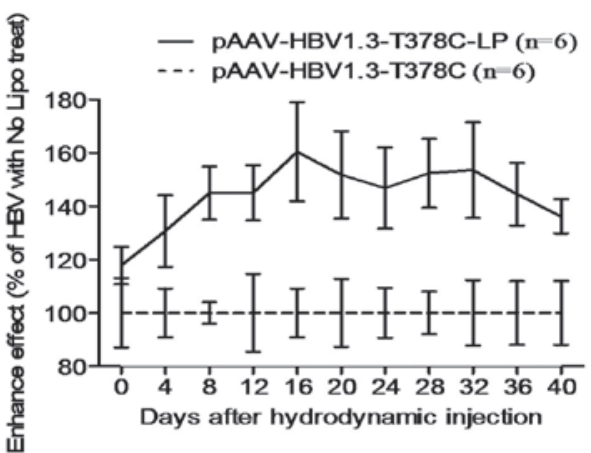

C
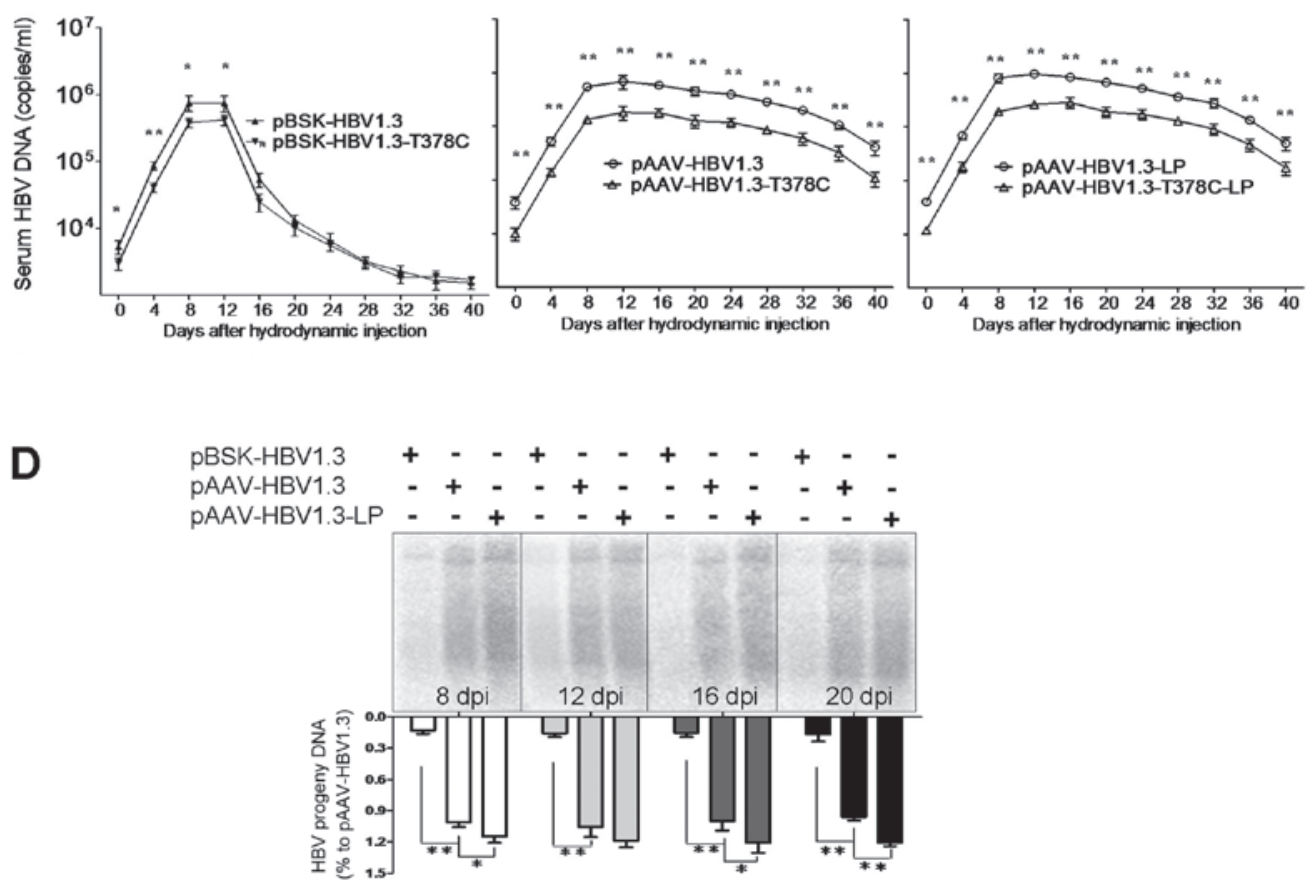

Figure 2. Replication of pBSK/pAAV-HBV1.3 and -T378C in the murine model. C57BL/6 (H-2b) mice were challenged with pBSK/pAAV-HBV1.3 and -T378C by tail vein administration of hydrodynamic injection. At the indicated time points, HBV DNA and proteins in the sera and liver samples were measured by qPCR targeted to HBV RC DNA, southern blot analysis, enzyme-linked immunosorbent assay and immunohistochemical analysis, respectively. (A-C) HBV DNA serum analysis of mice from the pBSK/pAAV-HBV1.3 and -T378C groups by qPCR. (A) Mice were divided into the wild-type and T378C groups. The kinetics of HBV replication expression of each group, which contained pBSK, pAAV and pAAV-Lipo subgroups. (B) The enhanced HBV replication effect of LP in vivo is shown as the upregulation of serum HBV DNA in LP-mixed mice compared with that of mice without LP. The average HBV DNA copy number of the unmixed control group at each of the indicated time points was set as 100\%. (C) The effect of T378C in vivo on the pBSK, pAAV and pAAV-Lipo subgroups is shown. (D) To detect HBV DNA in the liver, mice from each group were sacrificed at the indicated time points. Total DNA was isolated from the liver tissue and subjected to southern blot analysis. HBV, hepatitis B virus; LP, Lipofectamine 2000.

mice tail veins. C57BL/6 mice were respectively challenged with pBSK-HBV1.3, -HBV1.3-sM75T, pAAV-HBV1.3 and -HBV1.3-sM75T with or without LP by HI. HBV DNA in the mouse sera and liver samples were measured by qPCR targeted to RC and southern blot analysis at the indicated time points, respectively. Based on the in vitro results, LP significantly increased HBV replication in the sera (Fig. 2A and B) and liver (Fig. 2D) samples. The mouse model was analyzed 

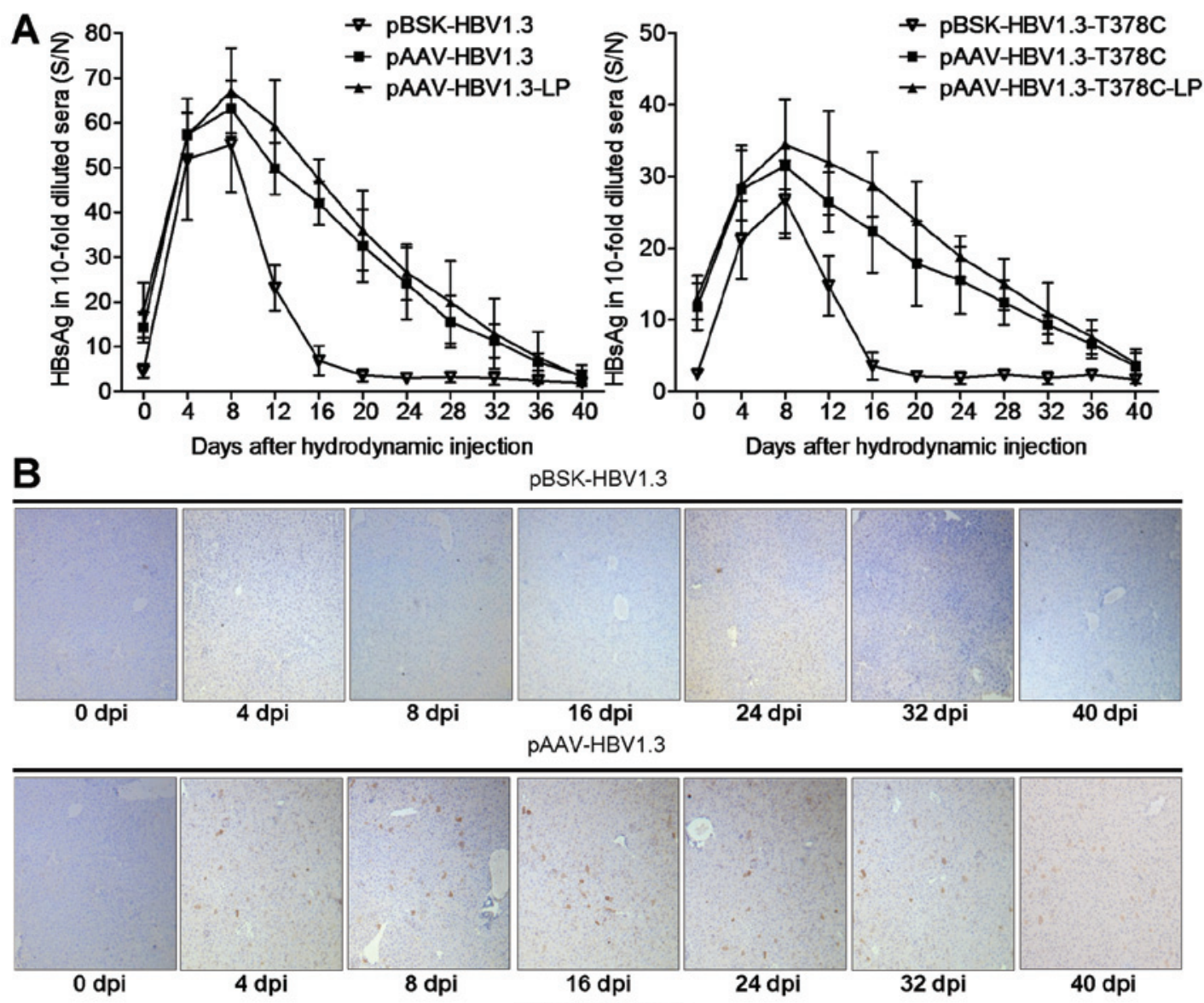

PAAV-HBV1.3-LP

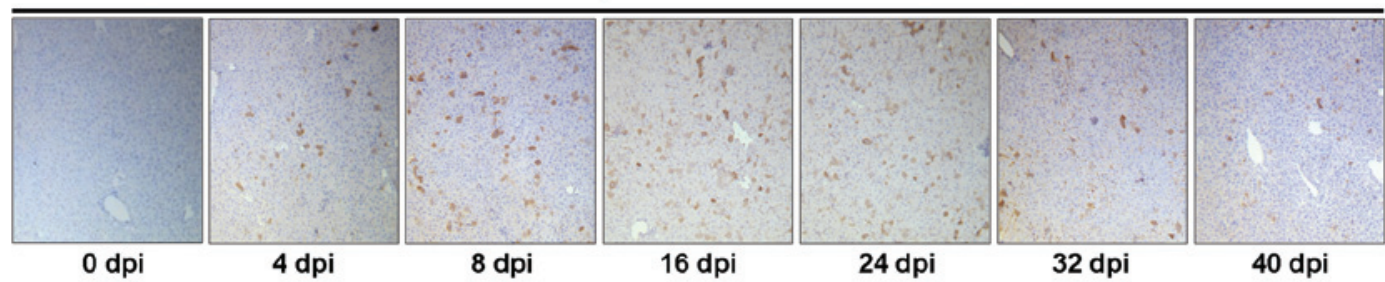

Figure 3. Kinetics of HBsAg and HBcAg expression. (A) The HBsAg level was determined using a commercial enzyme-linked immunosorbent assay kit and presented as the optical density value at $450 \mathrm{~nm}$. (B) Immunohistochemical staining of $\mathrm{HBcAg}$ in the mouse liver following administration of pBSK-HBV1.3, pAAV-HBV1.3 or pAAV-HBV1.3-LP. Magnification, x100. LP, Lipofectamine 2000; S/N, samples/negative.

following HI to confirm the results of our previous study. In line with our previous observations at the cellular level, the sM75T substitution also significantly depressed HBV replication in vivo at all time points (Fig. 2C), particularly in the pAAV and LP pAAV groups. Serum HBV DNA reached the highest level at 12 days postinfection (dpi) in all groups. By contrast, the pBSK group demonstrated a maximum viral DNA level at $12 \mathrm{dpi}$, which rapidly decreased thereafter. Moreover, on the background of the sM75T substitution, LP enhanced HBV replication.

$L P$ enhances $H B s A g$ and $H B c A g$ expression in mice. The HBV HBsAg level peaked at 8 dpi and then decreased gradually in mouse sera following HI with pBSK-HBV1.3/-sM75T or pAAV-HBV1.3/-sM75T (Fig. 3A), which was comparable with previously published data $(10,17)$. Serum HBsAg of the pAAV group remained positive for the complete observation period of 5 weeks, but only for $\sim 2$ weeks in the pBSK group.
Similar results were observed in the -sM75T group (Fig. 3A). The intrahepatic HBcAg was further analyzed by immunohistochemical staining with specific antibodies (Fig. 3C). HBcAg was expressed in hepatic cells in the pAAV-HBV1.3 group at 4 dpi and persisted for at least 5 weeks, particularly in the pAAV-HBV1.3-LP group, while no obvious HBcAg expression was identified in the pBSK-HBV1.3 group at any of the time points. $\mathrm{HBcAg}$ expression was not detected in livers of the mice that were administered with pAAV-HBV1.3-sM75T and pBSK-HBV1.3-sM75T via HI (data not shown).

\section{Discussion}

Approximately 2 billion people worldwide are infected with human HBV, of which $>350$ million are chronically infected and at high risk of progression to cirrhosis, liver failure or cancer. Over $50 \%$ of liver cancers worldwide are attributable to HBV infection. Thus, HBV-related liver diseases remain a 
major public health concern, with $\sim 1$ million mortalities annually. Individuals co-infected with HBV and HDV are at greater risk for rapid progression and severe disease.

LP is a proprietary formulation for transfecting nucleic acids (DNA, RNA and mRNA) into a wide range of eukaryotic cells and was used in our previous study to package HBV plasmid DNA and allowing entry into Huh7 cells (11). However, it is not clear whether LP promoted HBV replication when mixed and co-injected with pAAV-HBV1.3 into mice via the tail vein. According to the results of the present study, the pAAV vector was confirmed to be more suitable than pBSK for in vivo studies of $\mathrm{HBV}$ replication.

The HBV life cycle is a complicated process that is regulated by various host and viral factors $(21,22)$. Although the surface proteins predominantly assist viral envelopment and secretion, there are also studies that have indicated that the surface proteins may be involved in replication regulation (23). In our previous study, the T378C substitution led to sM75T within the HBsAg encoding gene and was identified to reduce cellular HBsAg expression and HBV replication (19), which was also demonstrated in our in vitro experiments. Thus, our previous and present results confirmed that surface proteins are not essential for HBV DNA replication in vivo and in vitro.

The in vivo HBV model based on the hydrodynamic injection of an engineered, replication-competent HBV DNA into the tail veins of C57BL/6 mice was used to evaluate the anti-HBV effect of nucleos(t)ide analog and study resistance mutation of HBV in vivo in our previous study (14). The greatest problem is that the HBV replication capacity in this mice model was too low for southern blot analysis. In the present study, we modified the mice model by injecting the mixture of LP and replication-competent HBV plasmid DNA. Based on our data, LP significantly enhanced the HBV replication capacity, the level of $\mathrm{HBsAg}$ and $\mathrm{HBcAg}$ in mouse liver. This improved model is more conducive to HBV study in vivo. In the future, we aim to achieve an improved standardization in vivo model and use it to perform HBV resistant phenotype analysis.

\section{Acknowledgements}

The authors would like to thank the participants for their work. This study was supported by the Qianjiang Talent Project of Zhejiang Province, China (grant no. 2012R10084).

\section{References}

1. Lavanchy D: Hepatitis B virus epidemiology, disease burden, treatment, and current and emerging prevention and control measures. J Viral Hepat 11: 97-107, 2004.

2. Lee WM: Hepatitis B virus infection. N Engl J Med 337: 1733-1745, 1997.
3. Yan $\mathrm{H}$, Zhong G, Xu G, et al: Sodium taurocholate cotransporting polypeptide is a functional receptor for human hepatitis B and D virus. Elife 1: e00049, 2012.

4. Paganelli M, Dallmeier K, Nyabi O, et al: Differentiated umbilical cord matrix stem cells as a new in vitro model to study early events during hepatitis B infection. Hepatology 57: 59-69, 2013.

5. Yan Z, Tan W, Dan Y, et al: Estrogen receptor alpha gene polymorphisms and risk of $\mathrm{HBV}$-related acute liver failure in the Chinese population. BMC Med Genet 13: 49, 2012.

6. Zoulim F and Locarnini S: Hepatitis B virus resistance to nucleos(t)ide analogues. Gastroenterology 137: 1593-1608, 2009.

7. Liu F, Chen L, Yu DM, et al: Evolutionary patterns of hepatitis B virus quasispecies under different selective pressures: correlation with antiviral efficacy. Gut 60: 1269-1277, 2011.

8. Takehara T, Suzuki T, Ohkawa K, et al: Viral covalently closed circular DNA in a non-transgenic mouse model for chronic hepatitis B virus replication. J Hepatol 44: 267-274, 2006.

9. Lim W, Kwon SH, Cho H, et al: HBx targeting to mitochondria and ROS generation are necessary but insufficient for HBV-induced cyclooxygenase-2 expression. J Mol Med (Berl) 88: 359-369, 2010.

10. Schinazi RF, Ilan E, Black PL, Yao X and Dagan S: Cell-based and animal models for hepatitis $\mathrm{B}$ and $\mathrm{C}$ viruses. Antivir Chem Chemother 10: 99-114, 1999.

11. Tang N, Huang AL, Zhang BQ, et al: Construction of recombinant eukaryotic expression plasmid containing 1.3-fold-overlength genome of HBV and its expression in HepG2 cells. Zhonghua Gan Zang Bing Za Zhi 11: 464-466, 2003 (In Chinese).

12. Huang LR, Wu HL, Chen PJ and Chen DS: An immunocompetent mouse model for the tolerance of human chronic hepatitis B virus infection. Proc Natl Acad Sci USA 103: 17862-17867, 2006.

13. Yin Y, Wu C, Song J, et al: DNA immunization with fusion of CTLA-4 to hepatitis B virus (HBV) core protein enhanced Th2 type responses and cleared $\mathrm{HBV}$ with an accelerated kinetic. PloS One 6: e22524, 2011.

14. Qin B, Budeus B, Cao L, et al: The amino acid substitutions rtP177G and rtF249A in the reverse transcriptase domain of hepatitis B virus polymerase reduce the susceptibility to tenofovir. Antiviral Res 97: 93-100, 2013.

15. Mizokami M, Orito E, Ohba K, Ikeo K, Lau JY and Gojobori T: Constrained evolution with respect to gene overlap of hepatitis $\mathrm{B}$ virus. J Mol Evol 44 (Suppl 1): S83-S90, 1997.

16. Gao $\mathrm{W}$ and $\mathrm{Hu} \mathrm{J}$ : Formation of hepatitis B virus covalently closed circular DNA: removal of genome-linked protein. J Virol 81: 6164-6174, 2007.

17. Miller RH, Kaneko S, Chung CT, Girones R and Purcell RH: Compact organization of the hepatitis B virus genome. Hepatology 9: 322-327, 1989.

18. Carman WF: The clinical significance of surface antigen variants of hepatitis B virus. J Viral Hepat 4 (Suppl 1): 11-20, 1997.

19. Qiu J, Qin B, Rayner S, et al: Novel evidence suggests Hepatitis B virus surface proteins participate in regulation of $\mathrm{HBV}$ genome replication. Virol Sin 26: 131-138, 2011.

20. Qin B, He T, Chen Z, Xu W, Pan G and Tu C: A novel method for the analysis of drug-resistant phenotypes of hepatitis B virus. Int J Mol Med 31: 975-981, 2013.

21. Hu J and Boyer M: Hepatitis B virus reverse transcriptase and epsilon RNA sequences required for specific interaction in vitro. J Virol 80: 2141-2150, 2006.

22. Hu J, Flores D, Toft D, Wang X and Nguyen D: Requirement of heat shock protein 90 for human hepatitis B virus reverse transcriptase function. J Virol 78: 13122-13131, 2004.

23. Chua PK, Wang RY, Lin MH, Masuda T, Suk FM and Shih C: Reduced secretion of virions and hepatitis B virus (HBV) surface antigen of a naturally occurring HBV variant correlates with the accumulation of the small $\mathrm{S}$ envelope protein in the endoplasmic reticulum and Golgi apparatus. J Virol 79: 13483-13496, 2005. 